\title{
Caracteristicas tomográficas de las lesiones pulmonares en pacientes hospitalizados con COVID-19 y su valor pronóstico.
}

\author{
María Montes de Oca ${ }^{1,2}$, Eleonora García ${ }^{1}$, Laura Sánchez-Traslaviña ${ }^{1}$, \\ Fátima Gutiérrez-Correia ${ }^{1}$, Irene Stulin ${ }^{1}$, Gabriela Blanco ${ }^{1}$, Isabel-Carlota Silva ${ }^{1}$, \\ Jennireth Quevedo ${ }^{1}$, María Cristina Arvelo ${ }^{1,2}$, Nathalia Valera ${ }^{1}$, Irene Papa ${ }^{1}$, \\ Santiago Bacci ${ }^{1}$, Fátima De Abreu ${ }^{1}$, Héctor Villarroel ${ }^{1}$, Juan Carlos Catari ${ }^{1}$, \\ José Luis Lope: ${ }^{1}$, Brigitte Moran ${ }^{1}$, Claudio Cárdenas ${ }^{1}$, Saverio Santucci ${ }^{1}$, \\ José Luis Viloria ${ }^{1}$, Jerry Gómes ${ }^{1}$, Antonio Martinelli ${ }^{1}$ y Manuel Guzmán ${ }^{1,2}$ \\ ${ }^{1}$ Hospital Centro Médico de Caracas, Caracas, Venezuela. \\ ${ }^{2}$ Universidad Central de Venezuela, Facultad de Medicina, Caracas, Venezuela.
}

Palabras clave: COVID-19; SARS-CoV-2; tomografía; mortalidad.

Resumen. La gravedad de las imágenes en la tomografía (TC) de tórax en pacientes con COVID-19 puede tener valor pronóstico. Este estudio evalúa el tipo, gravedad y frecuencia de las lesiones pulmonares de pacientes hospitalizados con COVID-19 y las diferencias en las características clínicas y desenlaces intrahospitalarios según la gravedad tomográfica. Se trata de un estudio observacional (cohorte retrospectiva) de pacientes hospitalizados con COVID-19. Se usó el formulario de ISARIC-OMS para recopilar datos. Se determinó el tipo de lesiones pulmonares, lóbulos afectados y puntuación de gravedad total en la TC de ingreso. Se calcularon el primer, segundo y tercer cuartiles de la puntuación total, para dividir la muestra en cuatro partes iguales (Q1, Q2, Q3 y Q4). Se incluyeron 556 pacientes, 336 hombres $(60,4 \%)$ y 220 mujeres $(39,6 \%)$, con edad promedio $61,9 \pm 15,8$ años y 532 tenían TC al ingreso. Los pacientes en los cuartiles más graves tenían más días de evolu-

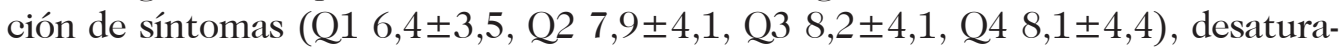

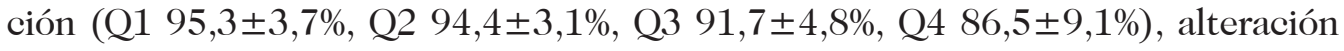
de marcadores inflamatorios, días de hospitalización (Q1 6,4 2,9, Q2 7,4 44,1 , Q3 9,6 $\pm 5,8$, Q4 13,1 $\pm 10,4)$, admisión a UCI (Q1-2,5\%, Q2-5,8\%, Q3-12,5\%, Q449,1\%), mortalidad (Q1-3,8\%, Q2-4,5\%, Q3-9,4\%, Q4-33,3\%), lesiones combinadas (vidrio deslustrado-consolidado) en la TC, opacidades lineales, patrón-empedrado, halo-invertido y bronquiectasia. La puntuación de la TC se correlacionó significativamente con el recuento de leucocitos, neutrófilos, linfocitos y otros marcadores inflamatorios. La evaluación semicuantitativa del compromiso pulmonar en la TC de tórax, puede ayudar a establecer la gravedad y predecir desenlaces clínicos en pacientes con COVID-19.

Autor de correspondencia: María Montes de Oca. Hospital Centro Médico de Caracas, Caracas, Venezuela. Correo electrónico: montesdeoca.maria@gmail.com 


\title{
Tomographic characteristics of lung lesions in hospitalized patients with COVID-19 and its prognostic value.
}

\author{
Invest Clin 2021; 62 (4): 357-370
}

Key words: COVID-19; SARS-CoV-2; chest tomography; mortality.

\begin{abstract}
The severity of lung involvement on chest tomography (CT) images in COVID-19 patients may have a prognostic value. This study assesses the type, severity and frequency of the different images of lung CT in hospitalized patients with COVID-19, and the differences in clinical characteristics and in-hospital outcomes, according to the CT severity score. This represents an observational study (retrospective cohort) of hospitalized patients with COVID-19. The ISARIC-WHO form was used to collect data. The type of lung lesions, affected lobes, and total CT severity score were determined at hospital admission. The first, second and third quartiles of the total CT score were calculated to divide the sample into four equal parts (Q1, Q2, Q3 and Q4). A total of 556 patients were included, 336 men (60.4\%) and 220 women (39.6\%), with a mean age of $61.9 \pm 15.8$ years; and 532 of them had CT scan at admission. Patients in the more severe quartiles had more days of symptoms evolution (Q1

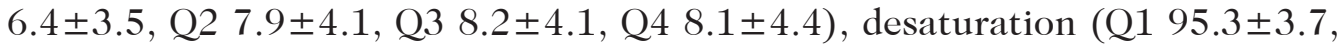
Q2 $94.4 \pm 3.1 \%$, Q3 91.7 $\pm 4.8 \%$, Q4 $86.5 \pm 9.1 \%$ ), alterations of inflammatory

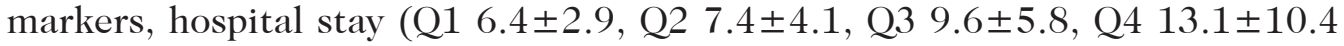
days), admission to ICU (Q1-2.5\%, Q2-5.8\%, Q3-12.5\%, Q4-49.1\%), mortality (Q1-3.8\%, Q2-4.5\%, Q3-9.4\%, Q4-33.3\%), mixed CT lesions (ground glass opacity-consolidation), linear opacities, crazy-paving pattern, reverse halo sign, and bronchiectasis. The total CT score significantly correlated with leukocyte, neutrophil and lymphocyte counts, and with other inflammatory markers. Semiquantitative evaluation of pulmonary involvement in the initial chest CT can help to establish the severity of the case and predict relevant clinical outcomes in COVID-19 patients.
\end{abstract}

Recibido: 05-09-2021 Aceptado: 26-10-2021

\section{INTRODUCGIÓN}

La enfermedad por coronavirus 2019 (COVID-19), causada por el nuevo virus SARS CoV-2, es una nueva enfermedad infecciosa que se convirtió rápidamente en una pandemia. Aunque la mayoría de las personas con esta infección evolucionan con una enfermedad leve-moderada o sin complicaciones, también se observa que una proporción importante de pacientes desarrollan enfermedad grave, que requiere hospitalización e ingreso a la unidad de cuidados intensivos (UCI). Los resultados de estudios previos informan que aproximadamente entre 10 y $17 \%$ de los pacientes hospitalizados por COVID-19 ameritaron ingreso en unidades de alta dependencia o UCI y la tasa de mortalidad intrahospitalaria varió entre 10 y $26 \%(1-3)$.

La tomografía computarizada (TC) de tórax es una herramienta ampliamente usa- 
da en la evaluación diaǵnóstica de rutina de los pacientes con neumonías en general, por ser relativamente fácil de realizar y permitir un diagnóstico rápido. En pacientes con infección por SARS-CoV-2, ésta se ha convertido en un estudio de gran importancia, no solo para diaǵnosticar la enfermedad COVID-19, sino también para estratificar su gravedad, monitorear la progresión de la enfermedad, evaluar la eficacia terapéutica y establecer pronóstico.

Los primeros estudios sobre hallazgos en la TC de tórax en COVID-19 indicaron como características típicas, la presencia de opacidades de aspecto de vidrio deslustrado (VD) con o sin consolidados, de distribución bilateral, posteriores y periféricas. Sin embargo, posteriormente, se han reportado otros tipos de imágenes tomográficas asociadas a COVID-19, incluyendo el patrón de pavimentación o empedrado, el signno del halo invertido, bronquiectasias retráctiles, engrosamientos lineales, consolidación multifocal en parches, entre otros (4).

Varios estudios han evaluado las características de las imágenes tomográficas en pacientes hospitalizados con el diagnóstico de COVID-19 y su relación con las condiciones clínicas y los desenlaces de los pacientes (5-10). Francone y colaboradores informaron que en pacientes sintomáticos con infección por SARS-CoV-2, la puntuación de la TC de tórax fue significativamente más alta en la categoría más grave de pacientes y que una puntuación $\geq 18$ de 25 puntos se asoció con mayor riesgo de mortalidad a corto plazo (9). Otros estudios mostraron que la puntuación de gravedad de la TC de tórax es un factor predictor fiable de enfermedad grave y mortalidad en personas con neumonía por SARS-CoV-2 $(8,10)$.

Existe información local limitada sobre los hallazgos tomográficos en pacientes hospitalizados con COVID-19 y su relación con los principales desenlaces clínicos. Por tanto, nuestro estudio buscó determinar el tipo, gravedad y frecuencia de las lesiones tomo- gráficas en pulmón de pacientes hospitalizados con diaǵnóstico de COVID-19. También evaluó las diferencias en las principales características clínicas y los desenlaces según la gravedad de las lesiones tomográficas pulmonares de estos pacientes.

\section{PACIENTES Y MÉTODOS}

Este es un estudio observacional de cohorte retrospectiva de pacientes hospitalizados con el diaǵnóstico clínico de COVID-19 en el Hospital Privado Centro Médico de Caracas, Venezuela entre el 1 de marzo del 2020 y el 29 de mayo del 2021 (556 pacientes). El protocolo del estudio fue aprobado por el Comité de Bioética de la institución.

\section{Recolección de datos}

Para la recopilación de los resultados se utilizó el formulario de informe de caso en papel (versión CORE en español del 23 de abril del 2020), desarrollado por el estudio ISARIC y la Organización Mundial de la Salud (OMS), para su uso en investigaciones del protocolo "Clinical Characterization Protocol UK (CCP-UK)" (https:// isaric.tghn.org/COVID-19-CRF/) y con la información recogida del registro diario realizado por los médicos tratantes de los pacientes. Se recolectó la información sobre los datos clínicos de los pacientes desde el momento del ingreso a la institución. Durante la hospitalización se evaluaron las medidas de gravedad de la enfermedad, los resultados de las pruebas de laboratorio de rutina para COVID-19 y los estudios de tomografía de tórax al momento del inǵreso. Se realizó un registro del nivel de atención requerida del paciente (sala de hospitalización o UCI), así como la duración de la hospitalización y los detalles del alta o muerte intrahospitalaria.

Un total de 532 pacientes tenían TC de tórax al momento del ingreso hospitalario, y los datos se extrajeron después que los estudios fueron evaluados por el equipo de especialistas en imágenes. 


\section{Confirmación del diagnóstico de COVID-19}

La confirmación diagnóstica de COVID-19 se basó en una o más de las siguientes pruebas: 1) Detección de secuencias virales específicas mediante pruebas de amplificación de ácidos nucleicos (AAN), con el resultado positivo de la prueba reacción en cadena de la polimerasa por transcripción inversa en tiempo real (rRT-PCR) para SARS-CoV-2. 2), prueba de antígeno para SARS-CoV-2 que detecta ciertas proteínas que se encuentran en la parte externa del virus. 3) Pruebas serológicas de determinación de anticuerpos para SARS-CoV-2 (IgM o IgGG).

Las muestras de las vías respiratorias superiores (hisopados nasofaríngeos) fueron tomadas siguiendo las directrices estandarizadas por la Orǵanización Mundial de la Salud (OMS).

\section{Criterios de hospitalización}

El principal criterio de ingreso hospitalario para pacientes con COVID-19 fue la presencia de hipoxemia en aire ambiente $\left(\mathrm{FiO}_{2}, 0,21\right)$ y/o la presencia de infiltrados pulmonares compatibles con neumonía por COVID-19. Una cama de la UCI fue definida como aquella con la capacidad de proporcionar ventilación mecánica y monitoreo continuo de signos vitales, con personal de enfermería de cuidados críticos y supervisión de médicos intensivistas. El ingreso a la UCI fue reservado para pacientes con insuficiencia respiratoria aguda severa que requirieron oxigenoterapia con cánula nasal de alto flujo o ventilación mecánica.

\section{Categorización de la gravedad de la enfermedad COVID-19}

Para establecer la gravedad de la enfermedad se siguieron los criterios del National Institutes of Health (NIH) (www. covid19treatmentguidelines.nih.gov): a. Enfermedad leve: personas que tienen cualquiera de los signos y síntomas de COVID-19 (p.
Ej., fiebre, tos, dolor de garǵanta, malestar general, dolor de cabeza, dolor muscular) sin dificultad para respirar, disnea o imagen torácica anormal. b. Enfermedad Moderada: personas que tienen evidencia de enfermedad de las vías respiratorias inferiores por clínica o imagen y una saturación de oxígeno $\left(\mathrm{SpO}_{2}\right) \geq 94 \%$ aire ambiente. c. Enfermedad Grave: personas con frecuencia respiratoria $>30$ respiraciones por minuto, $\mathrm{SpO}_{2}<94 \%$ al aire ambiente, relación de presión parcial de oxígeno arterial/ fracción de oxígeno inspirado $\left(\mathrm{PaO}_{2} / \mathrm{FiO}_{2}\right)<300 \mathrm{mmHg}$, o infiltrados pulmonares $>50 \%$. d. Enfermedad Crítica: personas con insuficiencia respiratoria, shock séptico y/o disfunción de múltiples órganos.

\section{Protocolo de Tomografía}

Todos los estudios tomográficos fueron realizados con protocolos de baja dosis, con dos tomógrafos multidetectores (Brivo CT 38516 y Revolution EVO 64, General Electric) con una colimación de 400 (reconstrucción de $0,62 \mathrm{~mm}$ a $1,25 \mathrm{~mm}$ en ventana pulmonar y de $5 \mathrm{~mm}$ en ventana de mediastino). El tiempo de rotación del gantry varió de 0,4 s a $0,8 \mathrm{~s}$. Las tomografías de tórax se obtuvieron en decúbito supino desde el opérculo torácico hasta el hemiabdomen superior, en inspiración profunda y apnea, sin sincronización ECG. Se utilizaron bandas de modulación de dosis de órganos radiosensibles o superficiales. El voltaje de preferencia fue de $120 \mathrm{kVp}$ con selección automática basada en la contextura del paciente variando entre 80 mAs y $100 \mathrm{mAs}$ (DVP $<5 \mathrm{mS}$ ). En todos los casos se realizaron reconstrucciones multiplanares sagitales y coronales en ventana de parénquima.

Fueron implementadas todas las medidas de bioseguridad en las salas de tomografía, con uso de material de protección personal para enfermeras y técnicos radiólogos, así como posterior limpieza de la sala y del tomógrafo. 
Características y análisis semicuantitativo visual de las alteraciones pulmonares en la TC de tórax

Las TC de tórax fueron evaluadas para determinar las siguientes características: a) presencia de opacidad en VD; b) presencia de consolidados; c) número de lóbulos afectados donde estaban presentes estas lesiones; e) grado de afectación de cada lóbulo pulmonar, y la extensión global de la alteración pulmonar medida por una "puntuación de gravedad total". Las definiciones de los términos radiológicos como opacidad en VD, patrón de empedrado y consolidado pulmonar se basaron en el glosario estándar para imágenes torácicas publicado por la Sociedad Fleischner (11).

La puntuación de gravedad de la TC fue calculada visualmente según los criterios de Pan y colaboradores para cada uno de los cinco lóbulos pulmonares (12). Para el análisis se usó la siguiente escala de puntuación: 0 (sin compromiso), 1 (compromiso <5\%), 2 (compromiso de 5 a 25\%), 3 (compromiso de 26 a 50\%), 4 (compromiso de 51\% a 75\%), 5 (compromiso $>75 \%$ ). La puntuación total en la TC fue la resultante de la suma de cada puntuación lobular individual (rango de puntuación posible de 0 a 25).

Se calcularon el primer, segundo y tereer cuartiles con los datos de la puntuación total en la TC de tórax, para dividir la muestra en cuatro partes iguales o en cuatro grupos de pacientes. Para calcular los cuartiles, se introdujeron todos los datos de la puntuación total de la TC de tórax en una calculadora estadística de cuartiles. El primer cuartil (Q1) es aquel valor de la variable tal que la cuarta parte (25\%) de las observaciones son inferiores o iguales a él, y el resto (75\%) es superior o igual. El segundo cuartil (Q2) es la mediana, ya que se trata del valor localizado en la mitad de la distribución. El tercer cuartil es el valor tal que las tres cuartas partes de las observaciones son inferiores o iguales a él. Coinciden con los percentiles 25,50 y 75 respectivamente. Los valores para los cuartiles fueron: Q1 (0-8 puntos),
Q2 (9-12 puntos), Q3 (13-16 puntos) y Q4 (17-25 puntos).

\section{Análisis Estadístico}

La estadística descriptiva incluyó la frecuencia en número y porcentaje para las variables categóricas y la media más desviación estándar (DS) para las numéricas. Para comparar las variables cuantitativas entre los pacientes de los cuartiles de puntuación de gravedad de la TC de tórax se usó la prueba no paramétrica Kruskal-Wallis ANOVA y para las variables cualitativas la prueba Pearson Chi-cuadrado. La asociación entre la puntuación total de la TC de tórax y las pruebas de laboratorio se evaluó con la prueba no paramétrica de correlación Spearman Rank. Un valor de $\mathrm{p}$ de 0,05 o menos fue considerado estadísticamente siǵnificativo. Se utilizó el programa STATISTICA Versión 10 (StatSoft) para los análisis estadísticos.

\section{RESULTADOS}

Un total de 556 pacientes fueron incluidos en el estudio, 336 eran hombres $(60,4 \%)$ y 220 mujeres $(39,6 \%)$, con una edad promedio $61,9 \pm 15,8$ años. Entre ellos 16/556 $(2,9 \%)$ pacientes fueron trasladados a otras instituciones por lo que los detalles de los desenlaces (ingreso a la UCI, mortalidad y estancia hospitalaria) se analizaron en $\mathbf{5 4 0}$ pacientes. Las características clínicas generales y duración de la hospitalización del total de pacientes incluidos en el estudio se muestran en la Tabla I. Cerca del 50\% de los pacientes tenían 65 años o más, mientras que solo 2,5\% tenían 30 años o menos. Para el momento de su ingreso al hospital 66\% de los pacientes se encontraban en etapa temprana-progresiva de la evolución de los síntomas de la enfermedad (0-8 días), y 10,5\% en etapa tardía ( $\geq 14$ días) (Tabla I). Aproximadamente el 58\% de los pacientes ingresados tenían una enfermedad grave-crítica y menos del $4 \%$ enfermedad leve.

La distribución de los pacientes según los cuartiles de la puntuación de afectación 
pulmonar en la TC de tórax fue la siguiente: Q1 (0-8 puntos) 159 pacientes (29,9\%), Q2 (9-12 puntos) 137 (25,8\%), Q3 (13-16 puntos) $120(22,5 \%)$ y Q4 (17-25 puntos) 116 (21,8\%). La edad promedio y grupos etarios fueron similares entre los grupos de cuartiles tomográficos (Tabla I). La proporción de pacientes del sexo masculino, y aquellos con enfermedad grave-crítica, los días con síntomas previos al ingreso y de hospitalización, aumentaron a medida que empeoraba la gravedad de la alteración pulmonar en la TC de tórax (Q1 al Q4), mientras que la saturación a aire ambiente de ingreso disminuyó progresivamente (Tabla I).

Los valores de las pruebas de laboratorio y marcadores inflamatorios según los cuartiles tomográficos se muestran en la Tabla I. Los pacientes en los cuartiles Q3 y Q4 tenían valores más elevados al ingreso del recuento total de leucocitos, neutrófilos, plaquetas e índice Neutrófilos/Linfocitos (N/L) y valores más bajos de linfocitos. También se observaron niveles más elevados de proteína $\mathrm{C}$ reactiva (PCR), lactato deshidrogenasa (LDH), y Dímero D en los pacientes de estos cuartiles. La puntuación total de la extensión de las alteraciones pulmonares en la TC de Tórax se correlacionó significativamente de forma negativa con el recuento de linfocitos y directa con el recuento de leucocitos, neutrófilos y plaquetas, así como con los niveles de PCR, LDH, dímero D y ferritina (Tabla II).

Las Fig. 1 y la Tabla III muestran la puntuación de gravedad en la TC de tórax (total y por lóbulos), y la frecuencia de los tipos de lesiones pulmonares, respectivamente. El lóbulo con mayor puntuación de afectación fue el lóbulo inferior derecho, seguido del lóbulo inferior izquierdo. La mayoría de los pacientes presentaron solo imágenes de tipo VD $(56,7 \%)$, mientras que el $40 \%$ presentaron combinación de VD más consolidados y solo consolidados un porcentaje muy bajos de casos (2\%). La distribución periférica fue el patrón más frecuente $(77,8 \%)$, seguida de la difusa $(20,2 \%)$ y central solo en $2 \%$. La gran mayoría de los casos presentaron lesio- nes bilaterales $(92,5 \%)$ y $7,5 \%$ unilaterales. Las opacidades lineales y el patrón de empedrado se observaron en aproximadamente la mitad de los casos, mientras que las opacidades redondeadas y el signo de halo invertido en un tercio de los pacientes. Otro tipo de lesiones (bronquiectasias, adenopatías mediastinales y derrame pleural) fueron menos frecuentes. La Fig. 2 muestra algunas imágenes tomográficas de pacientes incluidos en el estudio que ilustran los principales hallazgos tomográficos de la enfermedad.

Los tipos de lesiones pulmonares en la TC de tórax según los cuartiles tomográficos se muestran en la Tabla III. La presencia de VD solo, fue más frecuente en el grupo de pacientes en los cuartiles bajos (Q1 y Q2), mientras que en los más altos (Q3 y Q4) se observó mayor proporción de pacientes con lesiones combinadas de VD más consolidados, así como la presencia de opacidades lineales, patrón de empedrado, halo invertido y bronquiectasias.

La frecuencia de pacientes con ingreso a la UCI y la mortalidad según los cuartiles tomográficos se muestran en las Figs. 3A y $3 \mathrm{~B}$, respectivamente. Se observó un incremento progresivo en el porcentaje de pacientes que ingresaron a la UCI (Figura $3 \mathrm{~A}$ ) y de la mortalidad (Fig. 3B) a medida que empeoraba la extensión de las lesiones pulmonares en la TC de tórax. Cerca de la mitad de los pacientes en el cuartil cuatro $(57 / 116)$ requirieron ingreso a la UCI y aproximadamente un tercio de los pacientes en este cuartil fallecieron $(36 / 110)$.

\section{DISCUSIÓN}

Los principales hallazgos de este trabajo referente a las características tomográficas pulmonares de los pacientes hospitalizados por COVID-19 fueron: Primero, las imágenes tomográficas tipo VD combinada o no con consolidado, de distribución periférica y bilateral fueron las más frecuentes. Otras imágenes comunes fueron las opacidades lineales y redondeadas, el patrón 


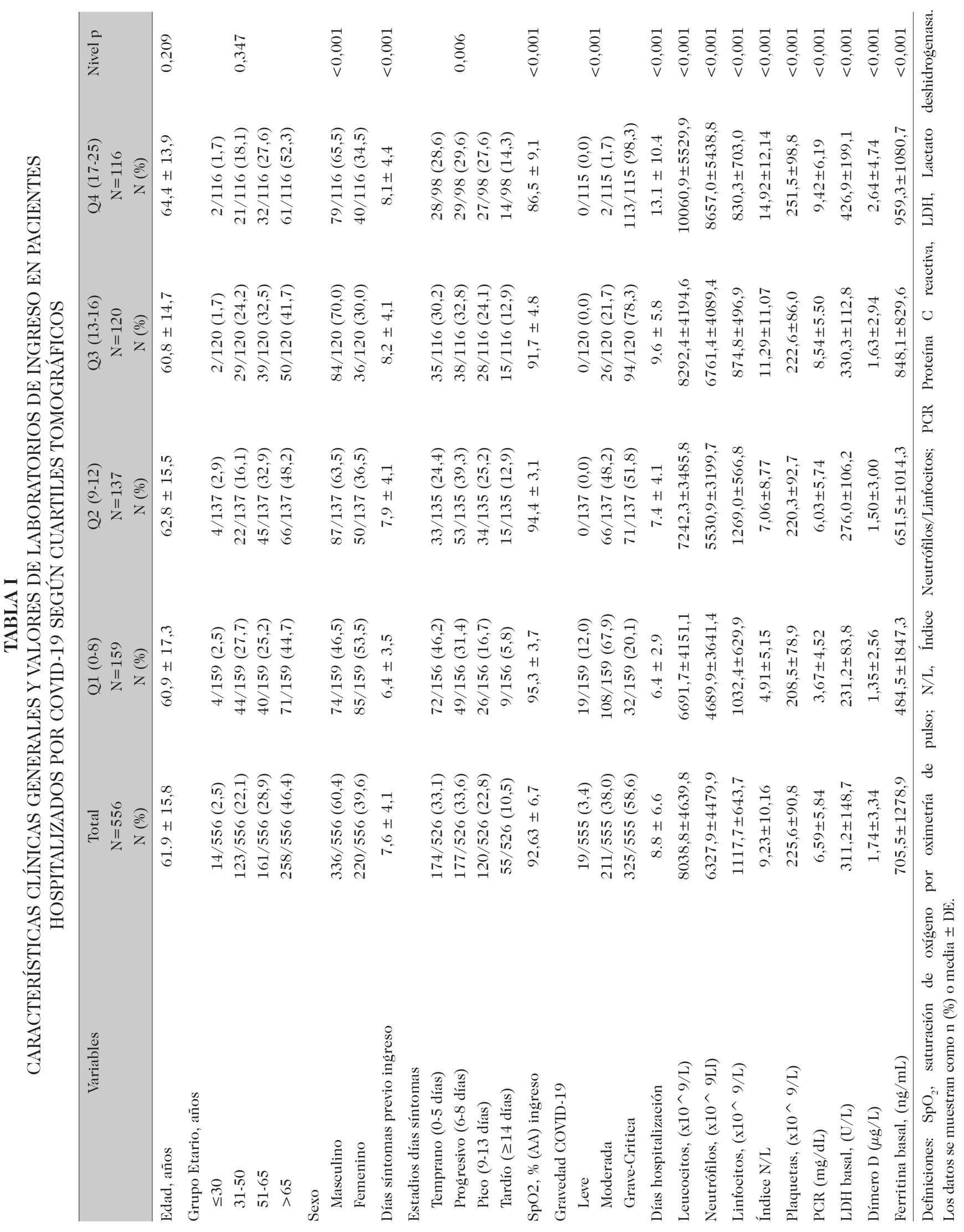


TABLA II

CORRELACIÓN DE SPEARMAN ENTRE PRUEBAS DE LABORATORIO Y PUNTUACIÓN TOTAL DE GRAVEDAD EN LA TC DE TÓRAX DE INGRESO.

\begin{tabular}{lcc}
\hline \multicolumn{1}{c}{ Variables } & Spearman $-\mathrm{R}$ & Nivel p \\
\hline Puntuación TC total- Leucocitos, basal, $\left(\mathrm{x} 10^{\wedge} \mathrm{9} / \mathrm{L}\right)$ & 0,348 & $<0,001$ \\
Puntuación TC total- Neutrófilos, basal, $\left(\mathrm{x} 10^{\wedge} 9 / \mathrm{L}\right)$ & 0,421 & $<0,001$ \\
Puntuación TC total- Linfocitos, basal, $\left(x 10^{\wedge} 9 / \mathrm{L}\right)$ & $-0,362$ & $<0,001$ \\
Puntuación TC total- Índice N/L & 0,497 & $<0,001$ \\
Puntuación TC total-Plaquetas, $\left(\mathrm{x} 10^{\wedge} 9 / \mathrm{L}\right)$ & 0,181 & $<0,001$ \\
Puntuación TC total- PCR basal, $(\mathrm{mg} / \mathrm{dL})$ & 0,489 & $<0,001$ \\
Puntuación TC total- LDH basal, $(\mathrm{U} / \mathrm{L})$ & 0,549 & $<0,001$ \\
Puntuación TC total- Dímero D basal, $(\mu \mathrm{g} / \mathrm{L})$ & 0,150 & $<0,001$ \\
Puntuación TC total- Ferritina basal, $(\mathrm{ng} / \mathrm{mL})$ & 0,454 & $<0,001$ \\
\hline
\end{tabular}

Definiciones: TC, Tomografía; PCR Proteína C reactiva, LDH, Lactato deshidrogenasa.

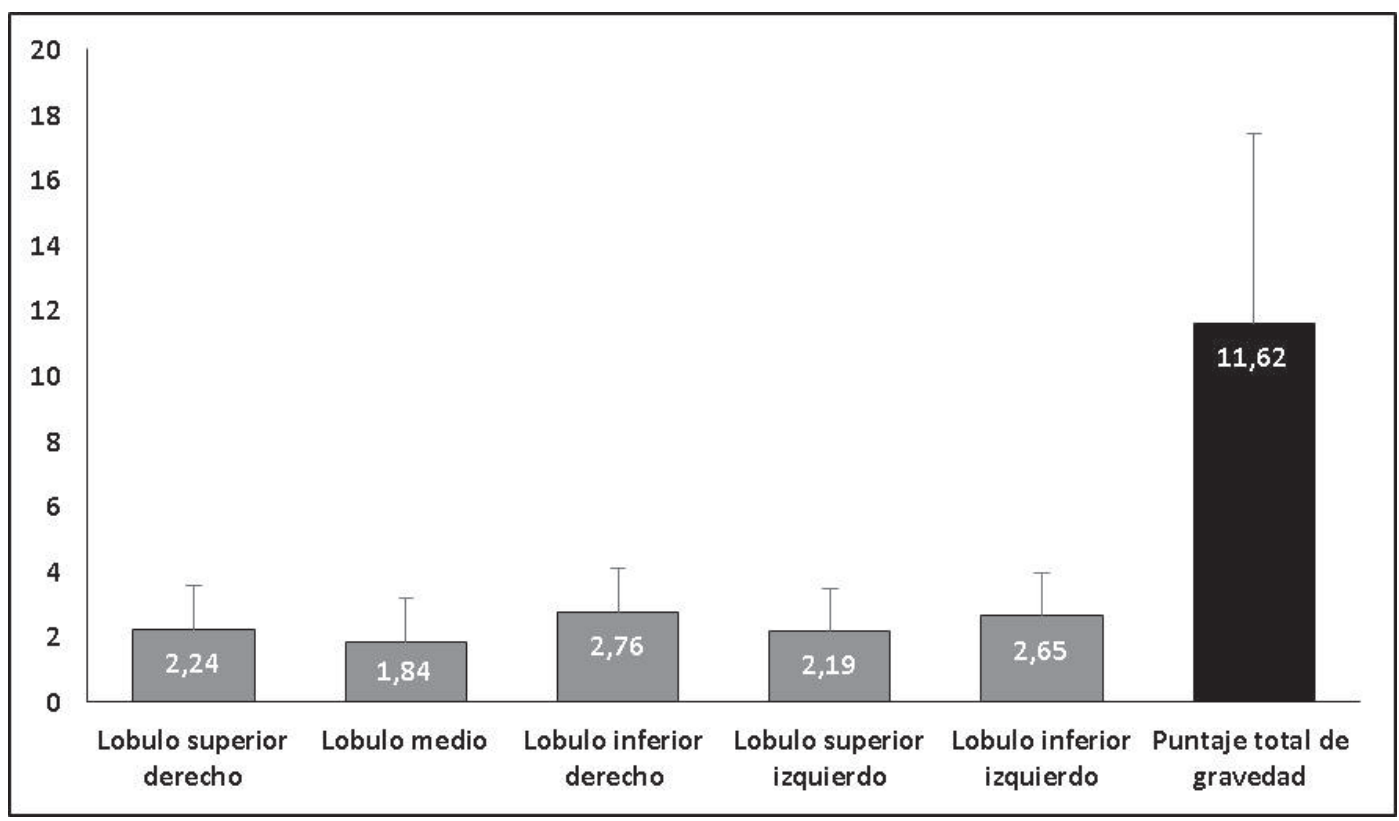

Fig. 1. Puntuación de la extensión de las lesiones pulmonares en la TC de tórax total y por lóbulos al inǵreso de pacientes hospitalizados por COVID-19. Los datos se muestran como media \pm DE.

de empedrado, el signo de halo invertido y las bronquiectasias retráctiles. Segundo, a medida que empeoraba la extensión de las imágenes tomográficas pulmonares en la TC de tórax, aumentaba el número de pacientes con enfermedad grave-critica, la proporción de pacientes masculinos, los días de síntomas previos, los días de hospitalización, los ingresos a la UCI y la mortalidad. Tereero, los pacientes con mayor puntuación de gravedad en la TC de tórax tenían valores más elevados de marcadores inflamatorios (leucocitos, neutrófilos e índice Neutrófilos/ Linfocitos, PCR, LDH, ferritina) y menor recuento de linfocitos. También se observó una correlación significativa entre la puntuación 


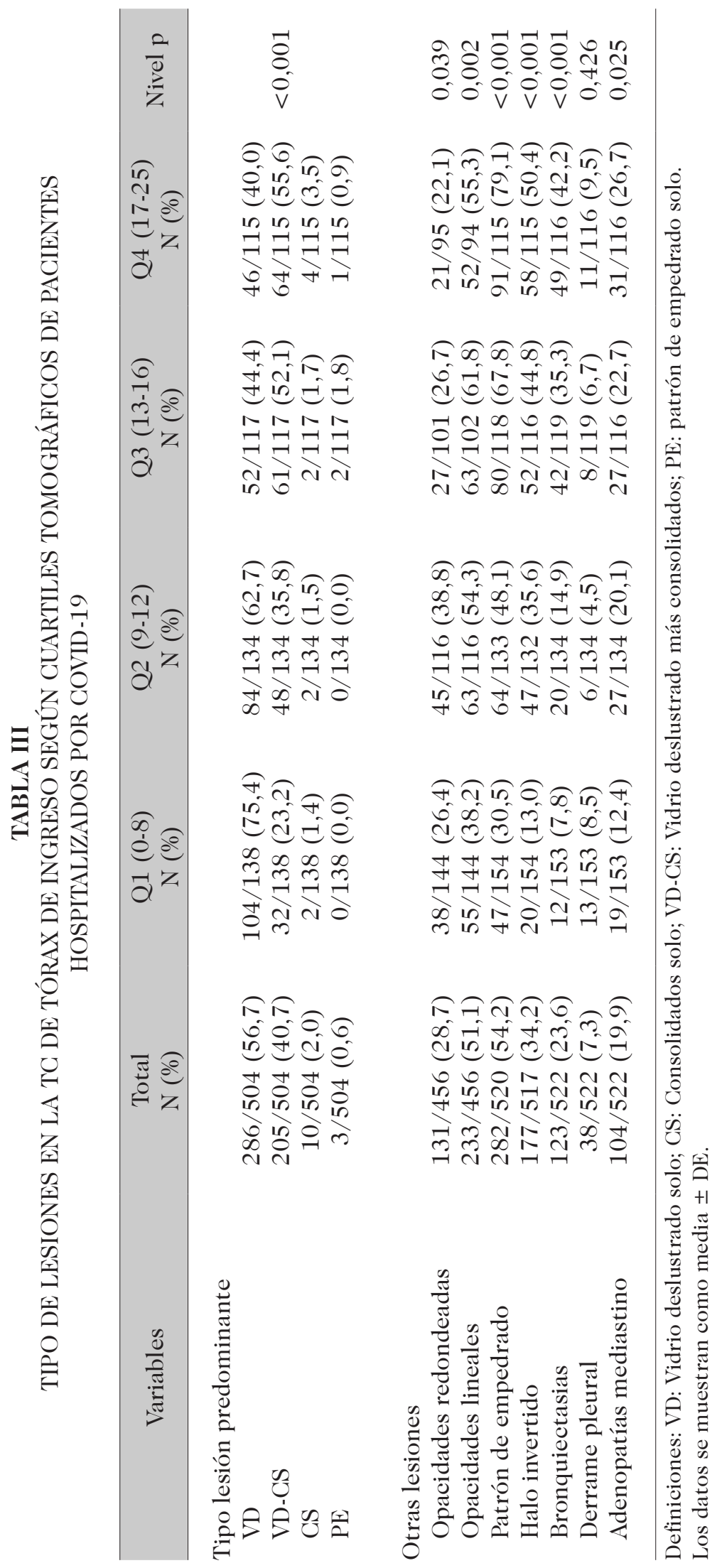




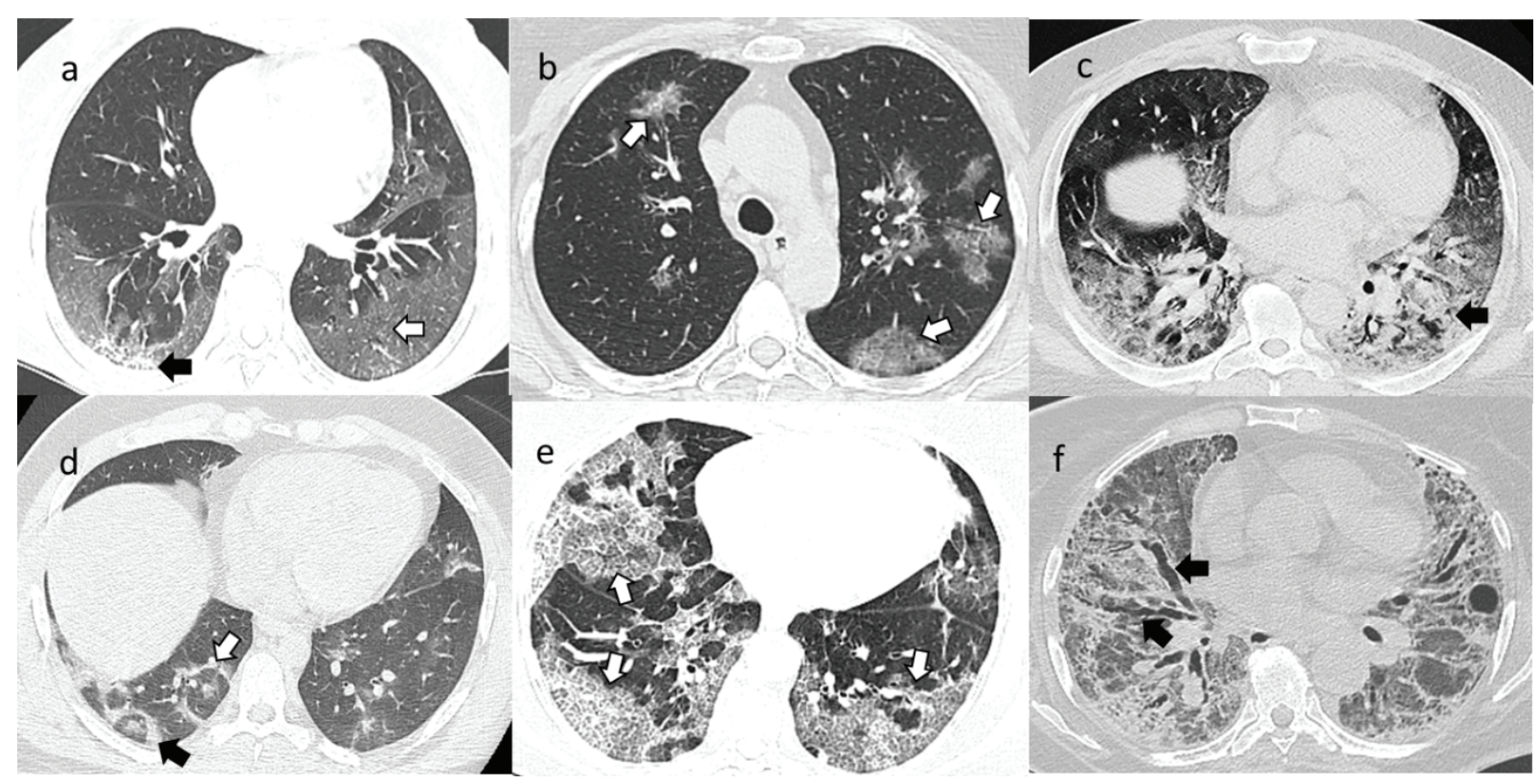

Fig. 2. Imáǵenes tomoǵráficas sin contraste en vista axial de lesiones pulmonares en pacientes con COVID-19. Hallazgoos en TC de tórax de neumonía por COVID-19 en imáǵenes axiales. Vidrio deslustrado (VD) de distribución periférica bilateral (a); VD con una morfología redondeada bilateral (b); Consolidados de distribución central y periférica bilateral (c); Halo invertido (área de opacificación débil en VD con un anillo de consolidación más densa) (d); patrón de empedrado (VD con engrosamiento de tabiques interlobulillar e intralobulillar superpuesto) (e); fibrosis pulmonar y bronquiectasia retráctil (f).

de gravedad en la TC de tórax y estas pruebas de laboratorio.

Los primeros estudios sobre alteraciones en la TC de tórax en pacientes con COVID-19 indican que la distribución bilateral de opacidades en VD con o sin consolidado, en los lóbulos inferiores, con distribución periférica, eran el sello cardinal de la COVID-19 (13-14). Sin embargo, análisis más detallados con el aumento de casos, mostraron otras características adicionales que incluían patrón de empedrado, engrosamientos septales, siǵno de halo invertido, bronquiectasias retráctiles, entre otros (15-17).

Las imágenes de la TC de tórax pueden manifestarse con características o patrones diferentes en pacientes con COVID-19 dependiendo de algunos factores, como el curso temporal y la gravedad de la enfermedad. Ye y colaboradores en un artículo de revisión muestran la frecuencia de las principales manifestaciones en la TC de tórax de pacientes con COVID-19 (4). Los hallazgos fueron los siguientes: VD (14 estudios) entre 14-98\% (promedio 64.2\%), consolidado (11 estudios) entre 2-64\% (promedio 39\%), VD más consolidado (5 estudios) entre 19-59\% (promedio 39,6\%), engrosamiento septal (5 estudios) entre 1-75\% (promedio 46,6\%), patrón reticular (5 estudios) entre 1-22\% (promedio 9,2\%), patrón en empedrado (5 estudios) entre 5-36\% (promedio 18,9\%), halo invertido (2 estudios) entre 2-3\% (promedio $2,5 \%$ ), bronquiectasia en 1 estudio $11 \%$, derrame pleural (5 estudios) entre 1-8\% (promedio 5,6\%), y adenopatías mediastinales (4 estudios) entre 4-8\% (promedio 6\%) (4).

Los resultados del presente estudio son consistentes con investigaciones anteriores y muestran que el VD es el tipo de lesión 

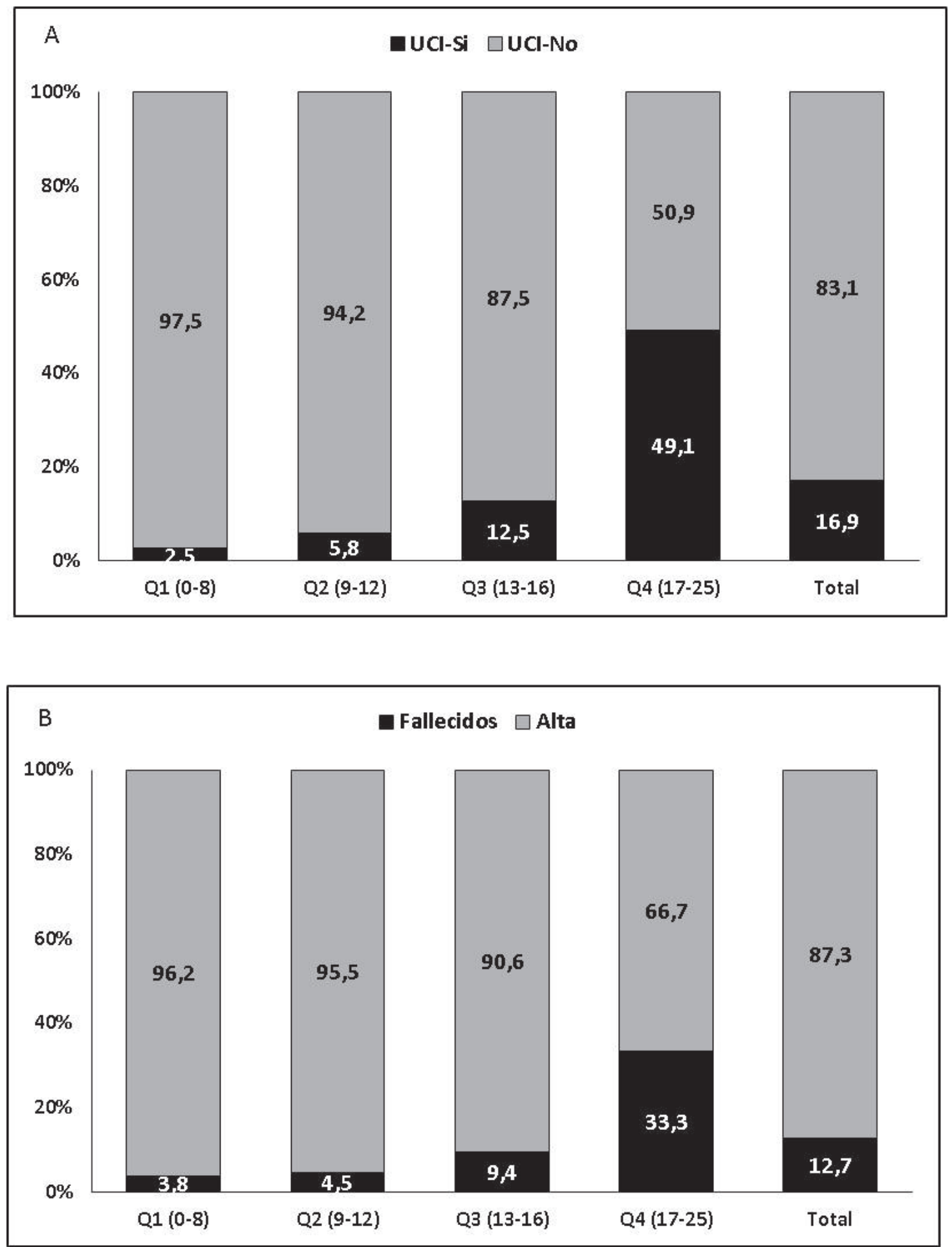

Figss. 3A y 3B. Ingresos a la UCI (A) y Mortalidad (B) en pacientes hospitalizados por COVID-19 según cuartiles tomográficos.

Los datos se muestran como n (\%).

*Pearson Chi-cuadrado p<0,001 (UCI-Si vs UCI-No, y Fallecidos vs Alta). 
más común (57\%), seguida de VD más consolidado en $40 \%$ de los casos, así como un predominio de distribución periférica en el $78 \%$ de los casos. También se observó mayor frecuencia de lesiones combinadas (VD, consolidado, opacidades lineales, patrón de empedrado, halo invertido y bronquiectasia) a medida que empeoraba la gravedad de la puntuación en la TC de tórax.

Se ha reportado que la gravedad de la puntuación en la TC de tórax puede ser un factor pronóstico para ingreso a la UCI y de mortalidad en pacientes con COVID-19 (4,6$9,18)$. Leger y colaboradores en un estudio retrospectivo en 80 pacientes evaluaron el valor de la TC para determinar el daño pulmonar en la neumonía por SARS-CoV-2, las características imagenológicas y la puntuación de gravedad (18). Las lesiones más frecuentes fueron VD (67\%), consolidado (25\%), patrón empedrado (11\%), derrame pleural, ganglios mediastínicos (5\%), y la distribución periférica (40\%) (18). También reportaron que la puntuación en la TG de tórax fue significativamente diferente entre los pacientes de bajo riesgo y alto riesgo (18). Usando el mismo método del presente estudio para evaluar la puntuación semicuantitativa en la TC de tórax (puntuación 0 a 25), otros autores encontraron en 130 pacientes sintomáticos con COVID-19 que una puntuación $\geq 18 / 25$ se asoció con mayor riesgo de mortalidad a corto plazo (24 días) y predecía mortalidad en el análisis univariado y multivariado (HR, 8,33; IC95\%, 3,19-21,73; HR, 3,74; 95\%IC, 1,10-12,77, respectivamente) (9). También encontraron que la puntuación en la TG se relacionó con los niveles de PCR y dímero D (9).

Otros estudios han evaluado el valor pronóstico de métodos diferentes de puntuación semicuantitativa en la TC de tórax (6). Charpentier y colaboradores en 210 pacientes con COVID-19, compararon la puntuación visual de gravedad en un rango de 0 a 20 utilizando áreas de consolidado solas $o$ asociadas con VD entre pacientes supervivientes y no supervivientes (6). La puntua- ción de gravedad en la TC fue significativamente mayor en los pacientes fallecidos, y el mejor punto de corte para distinguir entre supervivientes y no supervivientes fue 7/20 (6). La mortalidad a los 30 días en los pacientes con una puntuación $\geq 7$ fue $37,9 \%$ frente a $10,4 \%$ en aquellos con puntuación $<7$ (HR 3,16; IC95\% 1,50-6,43 independiente de la edad, frecuencia respiratoria, saturación de oxígeno, y comorbilidades) (6). El 50,5\% de los pacientes con puntuación $\geq 7$ fueron admitidos a la UCI comparado con $20,9 \%$ de aquellos con $<7$. Los niveles de dímero $\mathrm{D}$, hs-troponina I, los biomarcadores de inflamación, recuento de plaquetas y neutrófilos, fueron más altos, y el recuento de linfocitos más bajo, cuando la puntuación fue $\geq 7$ (6). Otros autores usando este mismo método de puntuación semicuantitativo (puntaje de gravedad de 0 a 20) encontraron que la puntuación en la TC fue el único predictor de mortalidad (OR 1,99, IC95\% $1,01-4,06)$ y un valor $\geq 7,5$ tenía la mayor sensibilidad y especificidad en la curva ROC para predecir la mortalidad (8). En Perú un estudio de cohorte retrospectivo en 254 pacientes con COVID-19 reportó que los hallazǵos tomográficos asociados a mortalidad fueron el patrón en empedrado (HR: 1,78; IC95\%: 1,03-3,06) y la mayor puntuación de gravedad (HR: 1,33; IC95\%: 1,20-1,48) (10). También proponen los valores de 7 (sensibilidad de $94,4 \%$ y especificidad de $100 \%$ ) y 13 (sensibilidad $84,9 \%$ y especificidad 70,6\%) como los puntos de cortes en el análisis de extensión de las lesiones pulmonares en la TC de tórax para identificar los casos con enfermedad moderada y grave, respectivamente $(10)$.

Nuestros resultados coinciden con los estudios anteriores y al igual que Francone y colaboradores, (quienes utilizaron el mismo sistema de puntuación de la TC de tórax) (9) muestran un incremento progresivo en los días de hospitalización, la admisión a la UCI y de la mortalidad intrahospitalaria a medida que se incrementó la puntuación de gravedad en la TC de tórax, con una tasa de 
mortalidad intrahospitalaria en los pacientes con mayor puntuación (Q4) del 33\% y de admisión a la UCI de 49\%. También van en línea con los estudios previos al mostrar mayores alteraciones en el recuento de la serie blanca y marcadores inflamatorios en los pacientes con mayor puntuación de gravedad en la TC de tórax. Estos resultados refuerzan las evidencias del valor pronóstico de la puntuación en la TC de tórax inicial en pacientes con COVID-19 y podrían ser de ayuda para estratificar la gravedad de los pacientes al momento de su ingreso hospitalario y especular sobre su pronóstico a corto plazo. También soportan que el análisis semicuantitativo visual de la TC de tórax simple estandarizado, que no requiere ningún software, es factible de realizar y proporciona una información valiosa y estructurada de los resultados de las imágenes pulmonares en estos pacientes.

El presente estudio tiene algunas limitaciones que deben ser comentadas. Primero, es un estudio observacional retrospectivo realizado durante un año de la pandemia de COVID-19, por lo que existen factores de confusión no medidos y se recogieron solo datos básicos, por lo que carecen del nivel de detalles necesarios para un análisis más extenso de aspectos, como el poder predictor de la TC de tórax sobre la mortalidad a mayor plazo. Nuestro análisis de supervivencia carece de datos de seguimiento más prolongados, y solo se limita a un período de observación de la estadía intrahospitalaria. Por otra parte, el diseño observacional del estudio, impide establecer relaciones causales. No obstante, estos resultados concuerdan con los datos reportados en otras series internacionales en pacientes hospitalizados con COVID-19 y representan el primer análisis en una muestra grande de pacientes sobre las imágenes en la TC de tórax en nuestra población. Segundo, nuestro centro es un hospital privado de atención terciaria en el área metropolitana de Caracas, lo que puede limitar la validez externa de los hallazgos a otro tipo de insti- tuciones de salud como las de atención pública o en el interior del país. Tercero, el estudio solo incluyó pacientes hospitalizados (principalmente pacientes con enfermedad moderada a grave-crítica), por lo tanto, no es posible generalizar estos hallazgos a pacientes no hospitalizados o con enfermedad menos grave.

En conclusión, los resultados del presente estudio pueden tener importantes implicaciones clínicas, al apoyar las evidencias de que una evaluación semicuantitativa visual de la extensión de las lesiones pulmonares en pacientes con COVID-19 en la TC de tórax inicial, pueden ayudar a determinar la gravedad de la enfermedad y predecir desenlaces clínicos relevantes, como el ingreso a la UCI o muerte intrahospitalaria en estos pacientes. La evaluación semicuantitativa visual es un análisis sencillo que puede ser útil para determinar conductas de rutina en el manejo de estos pacientes y debería ser incorporada en los algoritmos de estratificación de riesgo y en los informes estructurados de la TC de tórax por parte de los especialistas en imágenes.

\section{REFERENCIAS}

1. Petrilli CM, Jones SA, Yang J, Rajagopalan H, O'Donnell L, Chernyak Y, Tobin KA, Cerfolio RJ, Francois F, Horwitz LI. Factors associated with hospital admission and critical illness among 5279 people with coronavirus disease 2019 in New York City: prospective cohort study. BMJ. 2020;369:m1966.

2. Docherty AB, Harrison EM, Green CA, Hardwick HE, Pius R, Norman L, Holden KA, Read JM, Dondelinger F, Carson G, Merson L, Lee J, Plotkin D, Siǵfrid L, Halpin S, Jackson C, Gamble C, Horby PW, Nguyen-Van-Tam JS, Ho A, Russell CD, Dunning J, Openshaw PJ, Baillie JK, Semple MG; ISARIC4C investigators. Features of 20133 UK patients in hospital with covid-19 using the ISARIC WHO Clinical Characterisation Protocol: prospective observational cohort study. BMJ. 2020;369:m1985.

Vol. 62(4): 357 - 370, 2021 
3. Casas-Rojo JM, Antón-Santos JM, MillánNúñez-Cortés $J$, Lumbreras-Bermejo C, Ramos-Rincón JM, Roy-Vallejo E, ArteroMora A, Arnalich-Fernández F, García-Bruñén JM, Vargas-Núñez JA, Freire-Castro SJ, Manzano-Espinosa L, Perales-Fraile I, Crestelo-Viéitez A, Puchades-Gimeno F, Rodilla-Sala E, Solís-Marquínez MN, Bonet-Tur D, Fidalgo-Moreno MP, Fonseca-Aizpuru EM, Carrasco-Sánchez FJ, Rabadán-Pejenaute E, Rubio-Rivas $\mathrm{M}$, Torres-Peña JD, Gómez-Huelgas R; en nombre del Grupo SEMI-COVID-19 Network. Clinical characteristics of patients hospitalized with COVID-19 in Spain: Results from the SEMI-COVID-19 Registry. Rev Clin Esp (Bare) 2020;220:480-494.

4. Ye $Z$, Zhang $Y$, Wang $Y$, Huang $Z$, Song $B$. Chest CT manifestations of new coronavirus disease 2019 (COVID-19): a pictorial review. Eur Radiol 2020;30:4381-4389.

5. Colombi D, Bodini FC, Petrini M, Maffi G, Morelli N, Milanese G, Silva M, Sverzellati N, Michieletti E. Well-aerated Lung on Admitting Chest CT to Predict Adverse Outcome in COVID-19 Pneumonia. Radiology 2020;296:E86-E96.

6. Charpentier E, Soulat G, Fayol A, Hernigou $\mathrm{A}$, Livrozet $\mathrm{M}$, Grand T, Reverdito G, Al Haddad J, Dang Tran KD, Charpentier A, Clement O, Hulot JS, Mousseaux E. Visual lung damage CT score at hospital admission of COVID-19 patients and 30-day mortality. Eur Radiol 2021:1-10.

7. Li Y, Yang $Z$, Ai T, Wu S, Xia L. Association of "initial CT" findings with mortality in older patients with coronavirus disease 2019 (COVID-19). Eur Radiol 2020;30:61866193.

8. Tabatabaei SMH, Rahimi H, Moghaddas F, Rajebi H. Predictive value of CT in the short-term mortality of Coronavirus Disease 2019 (COVID-19) pneumonia in nonelderly patients: A case-control study. Eur J Radiol 2020;132:109298.

9. Francone M, Iafrate F, Masei GM, Coco S, Cilia F, Manganaro L, Panebianco V, Andreoli C, Colaiacomo MC, Zingaropoli MA, Ciardi MR, Mastroianni CM, Puǵliese F, Alessandri F, Turriziani $O$, Ricei $P$, Catalano C. Chest CT score in COVID-19 patients: correlation with disease severi- ty and short-term prognosis. Eur Radiol 2020;30:6808-6817.

10. Contreras-Grande J, Pineda-Borja V, Díaz H, Calderon-Anyosa RJC, Rodríguez B, Morón M. Hallazgoos tomográficos pulmonares asociados a severidad y mortalidad en pacientes con la COVID-19. Rev Peru Med Exp Salud Publica 2021;38(2).

11. Hansell DM, Bankier AA, MacMahon H, McLoud TC, Müller NL, Remy J. Fleischner Society: glossary of terms for thoracic imaǵing. Radiology 2008;246:697-722.

12. Pan F, Ye T, Sun P, Gui S, Liang B, Li L, Zheng D, Wang J, Hesketh RL, Yang L, Zheng C. Time course of lung changes at chest CT during recovery from Coronavirus Disease 2019 (COVID-19). Radiology 2020;295:715-721.

13. Wang D, Hu B, Hu C, Zhu F, Liu X, Zhang J, Wang $B$, Xiang $H$, Cheng $Z$, Xiong $Y$, Zhao Y, Li Y, Wang X, Peng Z. Clinical Characteristics of 138 hospitalized patients with 2019 novel coronavirus-Infected pneumonia in Wuhan, China. JAMA 2020;323:10611069.

14. Chung $M$, Bernheim A, Mei $X$, Zhang N, Huang M, Zeng X, Cui J, Xu W, Yang Y, Fayad ZA, Jacobi A, Li K, Li S, Shan H. CT Imaging features of 2019 novel coronavirus (2019-nCoV). Radiology 2020;295:202-207.

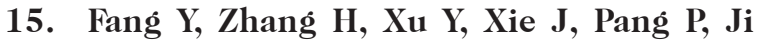
W. CT Manifestations of two cases of 2019 novel coronavirus (2019-nCoV) pneumonia. Radiology 2020;295:208-209.

16. Qian L, Yu J, Shi H. Severe acute respiratory disease in a Huanan seafood market worker: images of an early casualty. Radiol Cardiothorac Imaǵing 2020;2:e200033.

17. Bernheim $A$, Mei $X$, Huang $M$, Yang $Y$, Fayad ZA, Zhang N, Diao K, Lin B, Zhu X, Li K, Li S, Shan H, Jacobi A, Chung M. Chest CT findings in Coronavirus Disease-19 (COVID-19): relationship to duration of infection. Radiology 2020;295:200463.

18. Leger T, Jacquier A, Barral PA, Castelli M, Finance J, Lagier JC, Million M, Parola P, Brouqui P, Raoult D, Bartoli A, Gaubert JY, Habert P. Low-dose chest CT for diagnosing and assessing the extent of lung involvement of SARS-CoV-2 pneumonia using a semi quantitative score. PLoS One 2020;15:e241407. 